\title{
Revisiting Formic Acid Decomposition by a Graph-Theoretical Approach
}

Tomonori Ida ${ }^{*, \dagger}$ Manami Nishida,$^{\dagger}$ and Yuta Hori ${ }^{\ddagger}$

${ }^{\dagger}$ Graduate School of Natural Science and Technology, Kanazawa University, Kakuma,

Kanazawa 920-1192, Japan

†enter for Computational Sciences, University of Tsukuba, Tsukuba 305-8577, Japan

*Corresponding author E-mail: ida@se.kanazawa-u.ac.jp 
Figure S1. Unimolecular $\mathrm{HCOOH}$ decomposition including the oxygen atoms with a formal charge of -2 . To compare with Figure 3, the nodes added to Figure 3 are shown by yellow circles in this figure. 127 possible molecular graphs were reduced to 40 graphs in the reduction step.

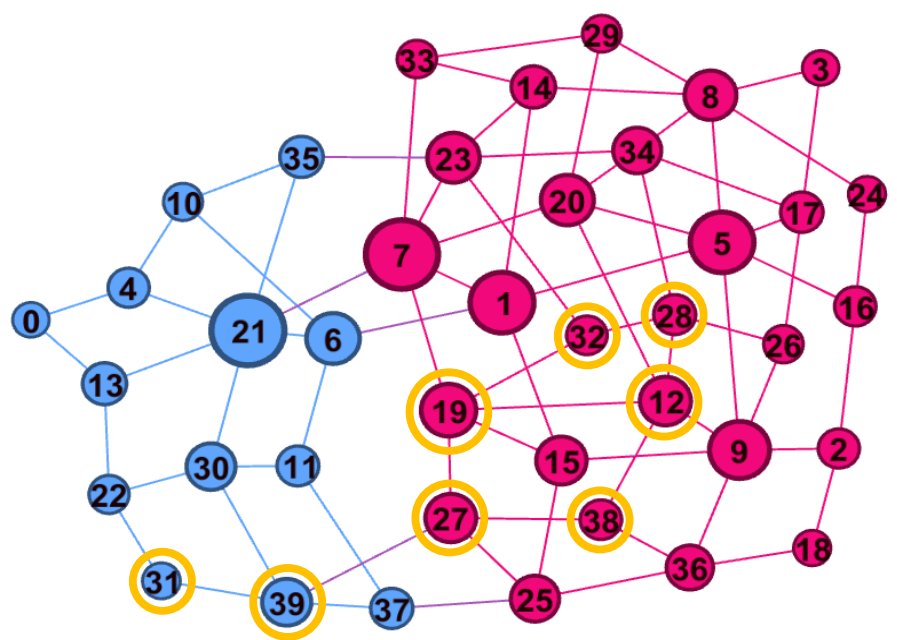


Table S1. Chemical formulae and connected information (CI) in the chemical reaction network of unimolecular $\mathrm{HCOOH}$ decomposition including the oxygen atoms with a formal charge of -2 . Formal charges $(\oplus$ and $\ominus$ ) are indicated on the right of the corresponding atom in the chemical formula.

\begin{tabular}{|c|c|c|}
\hline No. & Chemical formula & CI \\
\hline 0 & $\mathrm{CO}_{2}, \mathrm{H}_{2}$ & 4,13 \\
\hline 1 & $\mathrm{HC}(=\mathrm{O}) \mathrm{OH}$ & $5,6,7,14,15$ \\
\hline 2 & $\mathrm{CO}, \mathrm{H}_{2} \mathrm{O}$ & $9,16,18$ \\
\hline 3 & $\mathrm{C}(\mathrm{OH})_{2}$ & 8,17 \\
\hline 4 & $\mathrm{CO}_{2}, \mathrm{H}^{\oplus}, \mathrm{H}^{\ominus}$ & $0,10,21$ \\
\hline 5 & $\mathrm{C}^{\ominus}(=\mathrm{O}) \mathrm{OH}, \mathrm{H}^{\oplus}$ & $1,8,9,16,17,20$ \\
\hline 6 & $\mathrm{C}^{\oplus}(=\mathrm{O}) \mathrm{OH}, \mathrm{H}^{\ominus}$ & $1,10,11,21$ \\
\hline 7 & $\mathrm{HC}(=\mathrm{O}) \mathrm{O}^{\ominus}, \mathrm{H}^{\oplus}$ & $1,19,20,21,23,33$ \\
\hline 8 & $\mathrm{C}(\mathrm{OH}) \mathrm{O}^{\ominus}, \mathrm{H}^{\oplus}$ & $3,5,14,24,29,34$ \\
\hline 9 & $\mathrm{CO}, \mathrm{O} \ominus \mathrm{H}, \mathrm{H}^{\oplus}$ & $2,5,12,15,26,36$ \\
\hline 10 & $\mathrm{C}\left(=\mathrm{O}^{\oplus} \mathrm{H}\right)=\mathrm{O}, \mathrm{H}^{\ominus}$ & $4,6,35$ \\
\hline 11 & $\mathrm{C}\left(\equiv \mathrm{O}^{\oplus}\right) \mathrm{OH}, \mathrm{H}^{\ominus}$ & $6,30,37$ \\
\hline 12 & $\mathrm{CO}, \mathrm{O}^{2} \ominus, 2 \mathrm{H}^{\oplus}$ & $9,19,20,28,38$ \\
\hline 13 & $\mathrm{C}^{\oplus}(=\mathrm{O}) \mathrm{O}^{\ominus}, \mathrm{H}_{2}$ & $0,21,22$ \\
\hline 14 & $\mathrm{HC}^{\oplus}(\mathrm{OH}) \mathrm{O}^{\ominus}$ & $1,8,23,33$ \\
\hline 15 & $\mathrm{HC}^{\oplus}=\mathrm{O}, \mathrm{O} \ominus \mathrm{H}$ & $1,9,19,25$ \\
\hline 16 & $\mathrm{C}^{\ominus}(=\mathrm{O}) \mathrm{O}^{\oplus} \mathrm{H}_{2}$ & $2,5,24$ \\
\hline 17 & $\mathrm{C}^{\ominus}\left(=\mathrm{O}^{\oplus} \mathrm{H}\right) \mathrm{OH}$ & $3,5,26,34$ \\
\hline 18 & $\mathrm{C}^{\ominus} \equiv \mathrm{O}^{\oplus}, \mathrm{H}_{2} \mathrm{O}$ & 2,36 \\
\hline 19 & $\mathrm{HC}^{\oplus}=\mathrm{O}, \mathrm{O}^{2} \ominus, \mathrm{H}^{\oplus}$ & $7,12,15,27,32$ \\
\hline 20 & $\mathrm{C}^{\ominus}(=\mathrm{O}) \mathrm{O}^{\ominus}, 2 \mathrm{H}^{\oplus}$ & $5,7,12,29,34$ \\
\hline 21 & $\mathrm{C}^{\oplus}(=\mathrm{O}) \mathrm{O}^{\ominus}, \mathrm{H}^{\oplus}, \mathrm{H}^{\ominus}$ & $4,6,7,13,30,35$ \\
\hline 22 & $\mathrm{C}\left(\equiv \mathrm{O}^{\oplus}\right) \mathrm{O}^{\ominus}, \mathrm{H}_{2}$ & $13,30,31$ \\
\hline 23 & $\mathrm{HC}\left(=\mathrm{O}^{\oplus} \mathrm{H}\right) \mathrm{O}^{\ominus}$ & $7,14,32,34,35$ \\
\hline 24 & $\mathrm{C}\left(\mathrm{O}^{\oplus} \mathrm{H}_{2}\right) \mathrm{O}^{\ominus}$ & 8,16 \\
\hline 25 & $\mathrm{HC} \equiv \mathrm{O}^{\oplus}, \mathrm{O}^{\ominus} \mathrm{H}$ & $15,27,36,37$ \\
\hline 26 & $\mathrm{C}=\mathrm{O}^{\oplus} \mathrm{H}, \mathrm{O}^{\ominus} \mathrm{H}$ & $9,17,28$ \\
\hline 27 & $\mathrm{HC} \equiv \mathrm{O}^{\oplus}, \mathrm{O}^{2} \ominus, \mathrm{H}^{\oplus}$ & $19,25,38,39$ \\
\hline 28 & $\mathrm{C}=\mathrm{O}^{\oplus} \mathrm{H}, \mathrm{O}^{2} \ominus, \mathrm{H}^{\oplus}$ & $12,26,32,34$ \\
\hline 29 & $\mathrm{C}\left(\mathrm{O}^{\ominus}\right)_{2}, 2 \mathrm{H}^{\oplus}$ & $8,20,33$ \\
\hline 30 & $\mathrm{C}\left(\equiv \mathrm{O}^{\oplus}\right) \mathrm{O}^{\ominus}, \mathrm{H}^{\oplus}, \mathrm{H}^{\ominus}$ & $11,21,22,39$ \\
\hline 31 & $\mathrm{C}^{\oplus \equiv}=\mathrm{O}^{\oplus}, \mathrm{O}^{2} \ominus, \mathrm{H}_{2}$ & 22,39 \\
\hline 32 & $\mathrm{HC}^{\oplus}=\mathrm{O}^{\oplus} \mathrm{H}, \mathrm{O}^{2} \ominus$ & $19,23,28$ \\
\hline 33 & $\mathrm{HC}^{\oplus}(\mathrm{O} \ominus)_{2}, \mathrm{H}^{\oplus}$ & $7,14,29$ \\
\hline
\end{tabular}




\begin{tabular}{|c|c|c|}
\hline 34 & $\mathrm{C}^{\ominus}\left(=\mathrm{O}^{\oplus} \mathrm{H}\right) \mathrm{O}^{\ominus}, \mathrm{H}^{\oplus}$ & $8,17,20,23,28$ \\
\hline 35 & $\mathrm{C}^{\oplus}\left(=\mathrm{O}^{\oplus} \mathrm{H}\right) \mathrm{O}^{\ominus}, \mathrm{H}^{\ominus}$ & $10,21,23$ \\
\hline 36 & $\mathrm{C}^{\ominus} \equiv \mathrm{O}^{\oplus}, \mathrm{O}^{\ominus} \mathrm{H}, \mathrm{H}^{\oplus}$ & $9,18,25,38$ \\
\hline 37 & $\mathrm{C}^{\oplus} \equiv \mathrm{O}^{\oplus}, \mathrm{O} \ominus \mathrm{H}, \mathrm{H}^{\ominus}$ & $11,25,39$ \\
\hline 38 & $\mathrm{C}^{\ominus} \equiv \mathrm{O}^{\oplus}, \mathrm{O}^{2} \ominus, 2 \mathrm{H}^{\oplus}$ & $12,27,36$ \\
\hline 39 & $\mathrm{C}^{\oplus} \equiv \mathrm{O}^{\oplus}, \mathrm{O}^{2} \ominus, \mathrm{H}^{\oplus}, \mathrm{H}^{\ominus}$ & $27,30,31,37$ \\
\hline
\end{tabular}


Table S2. Chemical formulae, connected information (CI), and betweenness centrality (BC) values of molecular graphs in the chemical reaction network of $\mathrm{HCOOH}+\mathrm{H}_{2} \mathrm{O}$. Formal charges $\oplus$ and $\ominus$ are indicated on the right of the corresponding atom in the chemical formula. $\mathrm{BC}-\mathrm{c}$ and $\mathrm{BC}-\mathrm{h}$ are the $\mathrm{BC}$ values in the separation network for the decarboxylation and the dehydration groups, respectively.

\begin{tabular}{|c|c|c|c|c|c|}
\hline No. & Chemical formula & $\mathrm{CI}$ & $\mathrm{BC}$ & BC-c & BC-h \\
\hline 0 & $\mathrm{CO}_{2}, \mathrm{H}_{2} \mathrm{O}, \mathrm{H}_{2}$ & $6,16,27$ & 20.9 & 15.6 & \\
\hline 1 & $\mathrm{C}(=\mathrm{O})(\mathrm{OH})_{2}, \mathrm{H}_{2}$ & $7,12,28,30$ & 105.6 & 62.6 & \\
\hline 2 & $\mathrm{HC}(=\mathrm{O}) \mathrm{OH}, \mathrm{H}_{2} \mathrm{O}$ & $8,9,13,17,29,31$ & 669.0 & & 143.9 \\
\hline 3 & $\mathrm{CO}, 2 \mathrm{H}_{2} \mathrm{O}$ & $18,34,37$ & 66.8 & & 44.1 \\
\hline 4 & $\mathrm{HC}(\mathrm{OH})_{3}$ & $10,11,14,32$ & 115.0 & & 14.3 \\
\hline 5 & $\mathrm{C}(\mathrm{OH})_{2}, \mathrm{H}_{2} \mathrm{O}$ & $15,19,35,36$ & 74.2 & & 55.3 \\
\hline 6 & $\mathrm{CO}_{2}, \mathrm{H}_{2} \mathrm{O}, \mathrm{H}^{\oplus}, \mathrm{H}^{\ominus}$ & $0,20,22,26,39$ & 146.9 & 69.2 & \\
\hline 7 & $\mathrm{C}(=\mathrm{O})(\mathrm{OH})_{2}, \mathrm{H}^{\oplus}, \mathrm{H}^{\ominus}$ & $1,21,23,25,41,43$ & 336.5 & 145.1 & \\
\hline 8 & $\mathrm{C}(=\mathrm{O}) \mathrm{OH}, \mathrm{H}_{2} \mathrm{O}, \mathrm{H}^{\oplus}$ & $2,15,18,33,34,36,38,42$ & 667.2 & & 338.6 \\
\hline 9 & $\mathrm{C}^{\oplus}(=\mathrm{O}) \mathrm{OH}, \mathrm{H}_{2} \mathrm{O}, \mathrm{H}^{\ominus}$ & $2,21,22,24,39,43$ & 505.6 & 107.5 & \\
\hline 10 & $\mathrm{C} \ominus(\mathrm{OH})_{3}, \mathrm{H}^{\oplus}$ & $4,19,35,40$ & 68.5 & & 32.2 \\
\hline 11 & $\mathrm{C} \oplus(\mathrm{OH})_{3}, \mathrm{H}^{\ominus}$ & $4,23,41$ & 63.7 & 2.5 & \\
\hline 12 & $\mathrm{C}(=\mathrm{O})(\mathrm{OH}) \mathrm{O}^{\ominus}, \mathrm{H}_{2}, \mathrm{H}^{\oplus}$ & $1,25,44,46,60,72,74$ & 292.7 & 188.1 & \\
\hline 13 & $\mathrm{HC}(=\mathrm{O}) \mathrm{O}^{\ominus}, \mathrm{H}_{2} \mathrm{O}, \mathrm{H}^{\oplus}$ & $2,38,39,45,50,63,73$ & 668.5 & & 113.1 \\
\hline 14 & $\mathrm{HC}(\mathrm{OH})_{2} \mathrm{O}^{\ominus}, \mathrm{H}^{\oplus}$ & $4,40,41,47,61,75$ & 423.9 & & 84.2 \\
\hline 15 & $\mathrm{C}(\mathrm{OH}) \mathrm{O} \ominus, \mathrm{H}_{2} \mathrm{O}, \mathrm{H}^{\oplus}$ & $5,8,29,48,51,62,64,78,80$ & 563.2 & & 349.6 \\
\hline 16 & $\mathrm{CO}_{2}, \mathrm{O} \ominus \mathrm{H}, \mathrm{H}_{2}, \mathrm{H}^{\oplus}$ & $0,26,52,74$ & 41.2 & 31.6 & \\
\hline 17 & $\mathrm{HC}(=\mathrm{O}) \mathrm{OH}, \mathrm{O} \ominus \mathrm{H}, \mathrm{H}^{\oplus}$ & $2,42,43,53,56,63,75,82$ & 956.3 & & 201.8 \\
\hline 18 & $\mathrm{CO}, \mathrm{H}_{2} \mathrm{O}, \mathrm{O} \ominus \mathrm{H}, \mathrm{H}^{\oplus}$ & $3,8,31,54,59,68,83,87$ & 514.0 & & 304.3 \\
\hline 19 & $\mathrm{C}(\mathrm{OH})_{2}, \mathrm{O} \ominus \mathrm{H}, \mathrm{H}^{\oplus}$ & $5,10,32,57,64,85$ & 169.6 & & 106.7 \\
\hline 20 & $\mathrm{CO}_{2}, \mathrm{H}_{3} \mathrm{O}^{\oplus}, \mathrm{H}^{\ominus}$ & 6,77 & 7.1 & 3.2 & \\
\hline 21 & $\mathrm{C}(=\mathrm{O})\left(\mathrm{O}^{\oplus} \mathrm{H}_{2}\right) \mathrm{OH}, \mathrm{H}^{\ominus}$ & $7,9,65,79,84$ & 225.7 & 92.0 & \\
\hline 22 & $\mathrm{C}\left(=\mathrm{O}^{\oplus} \mathrm{H}\right)=\mathrm{O}, \mathrm{H}_{2} \mathrm{O}, \mathrm{H}^{\ominus}$ & $6,9,69,81$ & 83.6 & 35.4 & \\
\hline 23 & $\mathrm{C}\left(=\mathrm{O}^{\oplus} \mathrm{H}\right)(\mathrm{OH})_{2}, \mathrm{H}^{\ominus}$ & $7,11,66,86$ & 100.2 & 43.7 & \\
\hline 24 & $\mathrm{C}\left(\equiv \mathrm{O}^{\oplus}\right) \mathrm{OH}, \mathrm{H}_{2} \mathrm{O}, \mathrm{H}^{\ominus}$ & $9,67,70,88$ & 167.0 & 64.0 & \\
\hline 25 & $\mathrm{C}(=\mathrm{O})(\mathrm{OH}) \mathrm{O} \ominus, 2 \mathrm{H}^{\oplus}, \mathrm{H}^{\ominus}$ & $7,12,65,66,71,90,92$ & 491.6 & 229.9 & \\
\hline 26 & $\mathrm{CO}_{2}, \mathrm{O} \ominus \mathrm{H}, 2 \mathrm{H}^{\oplus}, \mathrm{H}^{\ominus}$ & $6,16,69,92$ & 91.3 & 43.9 & \\
\hline 27 & $\mathrm{C}^{\oplus}(=\mathrm{O}) \mathrm{O} \ominus, \mathrm{H}_{2} \mathrm{O}, \mathrm{H}_{2}$ & $0,39,44,49,74$ & 204.5 & 98.3 & \\
\hline
\end{tabular}




\begin{tabular}{|c|c|c|c|c|c|}
\hline 28 & $\mathrm{C}^{\oplus}(\mathrm{OH})_{2} \mathrm{O} \ominus, \mathrm{H}_{2}$ & $1,41,46,72$ & 85.1 & 31.5 & \\
\hline 29 & $\mathrm{HC}^{\oplus}(\mathrm{OH}) \mathrm{O} \ominus, \mathrm{H}_{2} \mathrm{O}$ & $2,15,47,50,73,75$ & 350.0 & & 147.5 \\
\hline 30 & $\mathrm{C} \oplus(=\mathrm{O}) \mathrm{OH}, \mathrm{O} \ominus \mathrm{H}, \mathrm{H}_{2}$ & $1,43,52,55,74$ & 243.8 & 111.6 & \\
\hline 31 & $\mathrm{HC}^{\oplus}=\mathrm{O}, \mathrm{H}_{2} \mathrm{O}, \mathrm{O} \ominus \mathrm{H}$ & $2,18,53,58,82$ & 301.8 & & 117.5 \\
\hline 32 & $\mathrm{HC}^{\oplus}(\mathrm{OH})_{2}, \mathrm{O}^{\ominus} \mathrm{H}$ & $4,19,56,75$ & 128.0 & & 54.1 \\
\hline 33 & $\mathrm{C}^{\ominus}(=\mathrm{O}) \mathrm{OH}, \mathrm{H}_{3} \mathrm{O}^{\oplus}$ & $8,48,54,76$ & 130.2 & & 82.5 \\
\hline 34 & $\mathrm{C} \ominus(=\mathrm{O}) \mathrm{O}^{\oplus} \mathrm{H}_{2}, \mathrm{H}_{2} \mathrm{O}$ & $3,8,51,83$ & 111.2 & & 68.6 \\
\hline 35 & $\mathrm{C} \ominus(\mathrm{OH})_{2} \mathrm{O} \oplus \mathrm{H}_{2}$ & $5,10,57,78$ & 51.8 & & 33.1 \\
\hline 36 & $\mathrm{C}^{\ominus}\left(=\mathrm{O}^{\oplus} \mathrm{H}\right) \mathrm{OH}, \mathrm{H}_{2} \mathrm{O}$ & $5,8,59,80,85$ & 144.6 & & 91.5 \\
\hline 37 & $\mathrm{C}^{\ominus} \equiv \mathrm{O}^{\oplus}, 2 \mathrm{H}_{2} \mathrm{O}$ & 3,87 & 5.1 & & 3.1 \\
\hline 38 & $\mathrm{C}^{\ominus}(=\mathrm{O}) \mathrm{O}^{\ominus}, \mathrm{H}_{2} \mathrm{O}, 2 \mathrm{H}^{\oplus}$ & $8,13,62,76,80,91$ & 303.7 & & 133.7 \\
\hline 39 & $\mathrm{C}^{\oplus}(=\mathrm{O}) \mathrm{O}^{\ominus}, \mathrm{H}_{2} \mathrm{O}, \mathrm{H}^{\oplus}, \mathrm{H}^{\ominus}$ & $6,9,13,27,65,67,77,81,92$ & 932.3 & 267.8 & \\
\hline 40 & $\mathrm{C}^{\ominus}(\mathrm{OH})_{2} \mathrm{O} \ominus, 2 \mathrm{H}^{\oplus}$ & $10,14,64,78,89$ & 198.2 & & 99.7 \\
\hline 41 & $\mathrm{C}^{\oplus}(\mathrm{OH})_{2} \mathrm{O}^{\ominus}, \mathrm{H}^{\oplus}, \mathrm{H}^{\ominus}$ & $7,11,14,28,66,79,90$ & 437.2 & 89.3 & \\
\hline 42 & $\mathrm{C}^{\ominus}(=\mathrm{O}) \mathrm{OH}, \mathrm{O}^{\ominus} \mathrm{H}, 2 \mathrm{H}^{\oplus}$ & $8,17,64,68,83,85,91$ & 478.2 & & 240.5 \\
\hline 43 & $\mathrm{C}^{\oplus}(=\mathrm{O}) \mathrm{OH}, \mathrm{O} \ominus \mathrm{H}, \mathrm{H}^{\oplus}, \mathrm{H}^{\ominus}$ & $7,9,17,30,69,70,84,86,92$ & 1051.1 & 266.4 & \\
\hline 44 & $\mathrm{C}(=\mathrm{O})\left(\mathrm{O}^{\oplus} \mathrm{H}_{2}\right) \mathrm{O}^{\ominus}, \mathrm{H}_{2}$ & $12,27,65,109$ & 101.0 & 66.2 & \\
\hline 45 & $\mathrm{HC}(=\mathrm{O}) \mathrm{O}^{\ominus}, \mathrm{H}_{3} \mathrm{O}^{\oplus}$ & $13,76,77,110$ & 178.7 & & 18.8 \\
\hline 46 & $\mathrm{C}\left(=\mathrm{O}^{\oplus} \mathrm{H}\right)(\mathrm{OH}) \mathrm{O}^{\ominus}, \mathrm{H}_{2}$ & $12,28,66,93,111$ & 111.1 & 65.8 & \\
\hline 47 & $\mathrm{HC}\left(\mathrm{O}^{\oplus} \mathrm{H}_{2}\right)(\mathrm{OH}) \mathrm{O}^{\ominus}$ & $14,29,78,79,94,112$ & 368.9 & & 76.5 \\
\hline 48 & $\mathrm{C}(\mathrm{OH}) \mathrm{O}^{\ominus}, \mathrm{H}_{3} \mathrm{O}^{\oplus}$ & $15,33,95,113$ & 74.0 & & 59.1 \\
\hline 49 & $\mathrm{C}\left(\equiv \mathrm{O}^{\oplus}\right) \mathrm{O}^{\ominus}, \mathrm{H}_{2} \mathrm{O}, \mathrm{H}_{2}$ & $27,67,96$ & 27.4 & 19.8 & \\
\hline 50 & $\mathrm{HC}\left(=\mathrm{O}^{\oplus} \mathrm{H}\right) \mathrm{O}^{\ominus}, \mathrm{H}_{2} \mathrm{O}$ & $13,29,80,81,97$ & 262.7 & & 35.8 \\
\hline 51 & $\mathrm{C}\left(\mathrm{O}^{\oplus} \mathrm{H}_{2}\right) \mathrm{O} \ominus, \mathrm{H}_{2} \mathrm{O}$ & $15,34,98,114$ & 79.6 & & 57.8 \\
\hline 52 & $\mathrm{C}\left(=\mathrm{O}^{\oplus} \mathrm{H}\right)=\mathrm{O}, \mathrm{O}^{\ominus} \mathrm{H}, \mathrm{H}_{2}$ & $16,30,69,111$ & 47.6 & 31.2 & \\
\hline 53 & $\mathrm{HC}(=\mathrm{O}) \mathrm{O}^{\oplus} \mathrm{H}_{2}, \mathrm{O}^{\ominus} \mathrm{H}$ & $17,31,83,84,112$ & 302.7 & & 58.6 \\
\hline 54 & $\mathrm{CO}, \mathrm{H}_{3} \mathrm{O}^{\oplus}, \mathrm{O} \ominus \mathrm{H}$ & $18,33,117$ & 81.6 & & 55.3 \\
\hline 55 & $\mathrm{C}\left(\equiv \mathrm{O}^{\oplus}\right) \mathrm{OH}, \mathrm{O} \ominus \mathrm{H}, \mathrm{H}_{2}$ & $30,70,96,115$ & 116.9 & 69.3 & \\
\hline 56 & $\mathrm{HC}\left(=\mathrm{O}^{\oplus} \mathrm{H}\right) \mathrm{OH}, \mathrm{O}^{\ominus} \mathrm{H}$ & $17,32,85,86,97,116$ & 390.0 & & 80.0 \\
\hline 57 & $\mathrm{C}\left(\mathrm{O}^{\oplus} \mathrm{H}_{2}\right) \mathrm{OH}, \mathrm{O}^{\ominus} \mathrm{H}$ & $19,35,98,118$ & 47.7 & & 36.7 \\
\hline 58 & $\mathrm{HC} \equiv \mathrm{O}^{\oplus}, \mathrm{H}_{2} \mathrm{O}, \mathrm{O} \ominus \mathrm{H}$ & $31,87,88,101$ & 166.3 & & 22.2 \\
\hline 59 & $\mathrm{C}=\mathrm{O}^{\oplus} \mathrm{H}, \mathrm{H}_{2} \mathrm{O}, \mathrm{O}^{\ominus} \mathrm{H}$ & $18,36,102,118$ & 58.2 & & 47.9 \\
\hline 60 & $\mathrm{C}(=\mathrm{O})(\mathrm{O} \ominus)_{2}, \mathrm{H}_{2}, 2 \mathrm{H}^{\oplus}$ & $12,71,93,119$ & 46.2 & 33.1 & \\
\hline 61 & $\mathrm{HC}\left(\mathrm{O}^{\ominus}\right)_{2} \mathrm{OH}, 2 \mathrm{H}^{\oplus}$ & $14,89,90,94,105,120$ & 411.8 & & 91.1 \\
\hline 62 & $\mathrm{C}\left(\mathrm{O}^{\ominus}\right)_{2}, \mathrm{H}_{2} \mathrm{O}, 2 \mathrm{H}^{\oplus}$ & $15,38,73,95,106,121$ & 225.7 & & 140.9 \\
\hline
\end{tabular}




\begin{tabular}{|c|c|c|c|c|c|}
\hline 63 & $\mathrm{HC}(=\mathrm{O}) \mathrm{O}^{\ominus}, \mathrm{O}^{\ominus} \mathrm{H}, 2 \mathrm{H}^{\oplus}$ & $13,17,91,92,97,120$ & 515.4 & & 73.4 \\
\hline 64 & $\mathrm{C}(\mathrm{OH}) \mathrm{O} \ominus, \mathrm{O} \ominus \mathrm{H}, 2 \mathrm{H}^{\oplus}$ & $15,19,40,42,75,98,106,123$ & 430.4 & & 272.0 \\
\hline 65 & $\mathrm{C}(=\mathrm{O})\left(\mathrm{O}^{\oplus} \mathrm{H}_{2}\right) \mathrm{O} \ominus, \mathrm{H}^{\oplus}, \mathrm{H}^{\ominus}$ & $21,25,39,44,100,122$ & 313.6 & 149.5 & \\
\hline 66 & $\mathrm{C}\left(=\mathrm{O}^{\oplus} \mathrm{H}\right)(\mathrm{OH}) \mathrm{O} \ominus, \mathrm{H}^{\oplus}, \mathrm{H}^{\ominus}$ & $23,25,41,46,100,107,124$ & 319.8 & 140.8 & \\
\hline 67 & $\mathrm{C}\left(\equiv \mathrm{O}^{\oplus}\right) \mathrm{O}^{\ominus}, \mathrm{H}_{2} \mathrm{O}, \mathrm{H}^{\oplus}, \mathrm{H}^{\ominus}$ & $24,39,49,99,108$ & 189.0 & 87.9 & \\
\hline 68 & $\mathrm{CO}, 2 \mathrm{O} \ominus \mathrm{H}, 2 \mathrm{H}^{\oplus}$ & $18,42,82,102,125$ & 194.6 & & 120.4 \\
\hline 69 & $\mathrm{C}\left(=\mathrm{O}^{\oplus} \mathrm{H}\right)=\mathrm{O}, \mathrm{O} \ominus \mathrm{H}, \mathrm{H}^{\oplus}, \mathrm{H}^{\ominus}$ & $22,26,43,52,104,124$ & 198.4 & 92.1 & \\
\hline 70 & $\mathrm{C}\left(\equiv \mathrm{O}^{\oplus}\right) \mathrm{OH}, \mathrm{O}^{\ominus} \mathrm{H}, \mathrm{H}^{\oplus}, \mathrm{H}^{\ominus}$ & $24,43,55,103,108,126$ & 325.3 & 141.3 & \\
\hline 71 & $\mathrm{C}(=\mathrm{O})\left(\mathrm{O}^{\ominus}\right)_{2}, 3 \mathrm{H}^{\oplus}, \mathrm{H}^{\ominus}$ & $25,60,107,128$ & 88.6 & 41.3 & \\
\hline 72 & $\mathrm{C}^{\oplus}(\mathrm{O} \ominus)_{2} \mathrm{OH}, \mathrm{H}_{2}, \mathrm{H}^{\oplus}$ & $12,28,90,93,109,119$ & 165.6 & 77.0 & \\
\hline 73 & $\mathrm{HC}^{\oplus}\left(\mathrm{O}^{\ominus}\right)_{2}, \mathrm{H}_{2} \mathrm{O}, \mathrm{H}^{\oplus}$ & $13,29,62,94,110,120$ & 299.3 & & 119.1 \\
\hline 74 & $\mathrm{C}^{\oplus}(=\mathrm{O}) \mathrm{O}^{\ominus}, \mathrm{O}^{\ominus} \mathrm{H}, \mathrm{H}_{2}, \mathrm{H}^{\oplus}$ & $12,16,27,30,92,96,111$ & 348.0 & 194.4 & \\
\hline 75 & $\mathrm{HC}^{\oplus}(\mathrm{OH}) \mathrm{O}^{\ominus}, \mathrm{O}^{\ominus} \mathrm{H}, \mathrm{H}^{\oplus}$ & $14,17,29,32,64,97,112,120$ & 538.9 & & 227.7 \\
\hline 76 & $\mathrm{C}^{\ominus}(=\mathrm{O}) \mathrm{O}^{\ominus}, \mathrm{H}_{3} \mathrm{O}^{\oplus}, \mathrm{H}^{\oplus}$ & $33,38,45,95,113$ & 132.2 & & 57.0 \\
\hline 77 & $\mathrm{C}^{\oplus}(=\mathrm{O}) \mathrm{O}^{\ominus}, \mathrm{H}_{3} \mathrm{O}^{\oplus}, \mathrm{H}^{\ominus}$ & $20,39,45,99$ & 175.6 & 36.4 & \\
\hline 78 & $\mathrm{C}^{\ominus}\left(\mathrm{O}^{\oplus} \mathrm{H}_{2}\right)(\mathrm{OH}) \mathrm{O} \ominus, \mathrm{H}^{\oplus}$ & $15,35,40,47,98,114,121$ & 343.6 & & 170.3 \\
\hline 79 & $\mathrm{C}^{\oplus}\left(\mathrm{O}^{\oplus} \mathrm{H}_{2}\right)(\mathrm{OH}) \mathrm{O}^{\ominus}, \mathrm{H}^{\ominus}$ & $21,41,47,100,122$ & 231.6 & 33.1 & \\
\hline 80 & $\mathrm{C}^{\ominus}\left(=\mathrm{O}^{\oplus} \mathrm{H}\right) \mathrm{O}^{\ominus}, \mathrm{H}_{2} \mathrm{O}, \mathrm{H}^{\oplus}$ & $15,36,38,50,113,123$ & 239.9 & & 109.4 \\
\hline 81 & $\mathrm{C}^{\oplus}\left(=\mathrm{O}^{\oplus} \mathrm{H}\right) \mathrm{O}^{\ominus}, \mathrm{H}_{2} \mathrm{O}, \mathrm{H}^{\ominus}$ & $22,39,50,100,124$ & 225.3 & 49.2 & \\
\hline 82 & $\mathrm{HC}^{\oplus}=\mathrm{O}, 2 \mathrm{O} \ominus \mathrm{H}, \mathrm{H}^{\oplus}$ & $17,31,68,101,116$ & 256.1 & & 100.3 \\
\hline 83 & $\mathrm{C}^{\ominus}(=\mathrm{O}) \mathrm{O}^{\oplus} \mathrm{H}_{2}, \mathrm{O}^{\ominus} \mathrm{H}, \mathrm{H}^{\oplus}$ & $18,34,42,53,98,118$ & 275.4 & & 144.1 \\
\hline 84 & $\mathrm{C}^{\oplus}(=\mathrm{O}) \mathrm{O}^{\oplus} \mathrm{H}_{2}, \mathrm{O}^{\ominus} \mathrm{H}, \mathrm{H}^{\ominus}$ & $21,43,53,103$ & 222.9 & 42.4 & \\
\hline 85 & $\mathrm{C}^{\ominus}(=\mathrm{O} \oplus \mathrm{H}) \mathrm{OH}, \mathrm{O}^{\ominus} \mathrm{H}, \mathrm{H}^{\oplus}$ & $19,36,42,56,102,118,123$ & 298.7 & & 145.4 \\
\hline 86 & $\mathrm{C}^{\oplus}(=\mathrm{O} \oplus \mathrm{H}) \mathrm{OH}, \mathrm{O} \ominus \mathrm{H}, \mathrm{H}^{\ominus}$ & $23,43,56,104,124$ & 276.1 & 50.8 & \\
\hline 87 & $\mathrm{C}^{\ominus} \equiv \mathrm{O}^{\oplus}, \mathrm{H}_{2} \mathrm{O}, \mathrm{O}^{\ominus} \mathrm{H}, \mathrm{H}^{\oplus}$ & $18,37,58,117,125$ & 185.9 & & 85.5 \\
\hline 88 & $\mathrm{C}^{\oplus} \equiv \mathrm{O}^{\oplus}, \mathrm{H}_{2} \mathrm{O}, \mathrm{O}^{\ominus} \mathrm{H}, \mathrm{H}^{\ominus}$ & $24,58,103,126$ & 119.4 & 10.7 & \\
\hline 89 & $\mathrm{C} \ominus(\mathrm{O} \ominus)_{2} \mathrm{OH}, 3 \mathrm{H}^{\oplus}$ & $40,61,106,121,127$ & 187.1 & & 103.3 \\
\hline 90 & $\mathrm{C}^{\oplus}(\mathrm{O} \ominus)_{2} \mathrm{OH}, 2 \mathrm{H}^{\oplus}, \mathrm{H}^{\ominus}$ & $25,41,61,72,107,122,128$ & 449.8 & 93.4 & \\
\hline 91 & $\mathrm{C}^{\ominus}(=\mathrm{O}) \mathrm{O}^{\ominus}, \mathrm{O} \ominus \mathrm{H}, 3 \mathrm{H}^{\oplus}$ & $38,42,63,106,123$ & 184.7 & & 79.7 \\
\hline 92 & $\mathrm{C}^{\oplus}(=\mathrm{O}) \mathrm{O}^{\ominus}, \mathrm{O}^{\ominus} \mathrm{H}, 2 \mathrm{H}^{\oplus}, \mathrm{H}^{\ominus}$ & $25,26,39,43,63,74,108,124$ & 844.9 & 247.6 & \\
\hline 93 & $\mathrm{C}\left(=\mathrm{O}^{\oplus} \mathrm{H}\right)\left(\mathrm{O}^{\ominus}\right)_{2}, \mathrm{H}_{2}, \mathrm{H}^{\oplus}$ & $46,60,72,107$ & 27.0 & 17.0 & \\
\hline 94 & $\mathrm{HC}\left(\mathrm{O}^{\ominus}\right)_{2} \mathrm{O}^{\oplus} \mathrm{H}_{2}, \mathrm{H}^{\oplus}$ & $47,61,73,121,122$ & 251.7 & & 40.7 \\
\hline 95 & $\mathrm{C}\left(\mathrm{O}^{\ominus}\right)_{2}, \mathrm{H}_{3} \mathrm{O}^{\oplus}, \mathrm{H}^{\oplus}$ & $48,62,76,110$ & 50.9 & & 36.9 \\
\hline 96 & $\mathrm{C}\left(\equiv \mathrm{O}^{\oplus}\right) \mathrm{O}^{\ominus}, \mathrm{O}^{\ominus} \mathrm{H}, \mathrm{H}_{2}, \mathrm{H}^{\oplus}$ & $49,55,74,108$ & 68.4 & 50.1 & \\
\hline 97 & $\mathrm{HC}\left(=\mathrm{O}^{\oplus} \mathrm{H}\right) \mathrm{O} \ominus, \mathrm{O}^{\ominus} \mathrm{H}, \mathrm{H}^{\oplus}$ & $50,56,63,75,123,124$ & 381.5 & & 56.0 \\
\hline
\end{tabular}




\begin{tabular}{|c|c|c|c|c|c|}
\hline 98 & $\mathrm{C}\left(\mathrm{O}^{\oplus} \mathrm{H}_{2}\right) \mathrm{O}^{\ominus}, \mathrm{O} \ominus \mathrm{H}, \mathrm{H}^{\oplus}$ & $51,57,64,78,83,112$ & 208.6 & & 130.7 \\
\hline 99 & $\mathrm{C}\left(\equiv \mathrm{O}^{\oplus}\right) \mathrm{O}^{\ominus}, \mathrm{H}_{3} \mathrm{O}^{\oplus}, \mathrm{H}^{\ominus}$ & 67,77 & 10.4 & 4.8 & \\
\hline 100 & $\mathrm{C}\left(=\mathrm{O}^{\oplus} \mathrm{H}\right)\left(\mathrm{O}^{\oplus} \mathrm{H}_{2}\right) \mathrm{O}^{\ominus}, \mathrm{H}^{\ominus}$ & $65,66,79,81$ & 92.8 & 41.3 & \\
\hline 101 & $\mathrm{HC} \equiv \mathrm{O} \oplus, 2 \mathrm{O} \ominus \mathrm{H}, \mathrm{H}^{\oplus}$ & $58,82,125,126$ & 137.2 & & 14.1 \\
\hline 102 & $\mathrm{C}=\mathrm{O}^{\oplus} \mathrm{H}, 2 \mathrm{O} \ominus \mathrm{H}, \mathrm{H}^{\oplus}$ & $59,68,85,116$ & 55.4 & & 38.5 \\
\hline 103 & $\mathrm{C}\left(\equiv \mathrm{O}^{\oplus}\right) \mathrm{O}^{\oplus} \mathrm{H}_{2}, \mathrm{O}^{\ominus} \mathrm{H}, \mathrm{H}^{\ominus}$ & $70,84,88$ & 51.8 & 20.3 & \\
\hline 104 & $\mathrm{C}(=\mathrm{O} \oplus \mathrm{H})_{2}, \mathrm{O} \ominus \mathrm{H}, \mathrm{H} \ominus$ & 69,86 & 6.7 & 2.5 & \\
\hline 105 & $\mathrm{HC}(\mathrm{O} \ominus)_{3}, 3 \mathrm{H}^{\oplus}$ & $61,127,128$ & 78.9 & & 5.7 \\
\hline 106 & $\mathrm{C}\left(\mathrm{O}^{\ominus}\right)_{2}, \mathrm{O}^{\ominus} \mathrm{H}, 3 \mathrm{H}^{\oplus}$ & $62,64,89,91,120$ & 172.2 & & 108.9 \\
\hline 107 & $\mathrm{C}\left(=\mathrm{O}^{\oplus} \mathrm{H}\right)\left(\mathrm{O}^{\ominus}\right)_{2}, 2 \mathrm{H}^{\oplus}, \mathrm{H}^{\ominus}$ & $66,71,90,93$ & 74.5 & 26.4 & \\
\hline 108 & $\mathrm{C}\left(\equiv \mathrm{O}^{\oplus}\right) \mathrm{O}^{\ominus}, \mathrm{O}^{\ominus} \mathrm{H}, 2 \mathrm{H}^{\oplus}, \mathrm{H}^{\ominus}$ & $67,70,92,96$ & 133.6 & 65.5 & \\
\hline 109 & $\mathrm{C}^{\oplus}\left(\mathrm{O}^{\ominus}\right)_{2} \mathrm{O}^{\oplus} \mathrm{H}_{2}, \mathrm{H}_{2}$ & $44,72,122$ & 43.1 & 16.6 & \\
\hline 110 & $\mathrm{HC}^{\oplus}\left(\mathrm{O}^{\ominus}\right)_{2}, \mathrm{H}_{3} \mathrm{O}^{\oplus}$ & $45,73,95$ & 47.5 & & 16.5 \\
\hline 111 & $\mathrm{C}^{\oplus}\left(=\mathrm{O}^{\oplus} \mathrm{H}\right) \mathrm{O}^{\ominus}, \mathrm{O}^{\ominus} \mathrm{H}, \mathrm{H}_{2}$ & $46,52,74,124$ & 106.6 & 50.4 & \\
\hline 112 & $\mathrm{HC}^{\oplus}\left(\mathrm{O}^{\oplus} \mathrm{H}_{2}\right) \mathrm{O}^{\ominus}, \mathrm{O}^{\ominus} \mathrm{H}$ & $47,53,75,98$ & 142.0 & & 56.2 \\
\hline 113 & $\mathrm{C} \ominus\left(=\mathrm{O}^{\oplus} \mathrm{H}\right) \mathrm{O}^{\ominus}, \mathrm{H}_{3} \mathrm{O}^{\oplus}$ & $48,76,80$ & 23.3 & & 13.4 \\
\hline 114 & $\mathrm{C}^{\ominus}\left(\mathrm{O}^{\oplus} \mathrm{H}_{2}\right)_{2} \mathrm{O}^{\ominus}$ & 51,78 & 8.6 & & 4.5 \\
\hline 115 & $\mathrm{C}^{\oplus} \equiv \mathrm{O}^{\oplus}, 2 \mathrm{O} \ominus \mathrm{H}, \mathrm{H}_{2}$ & 55,126 & 15.4 & 5.0 & \\
\hline 116 & $\mathrm{HC}^{\oplus}=\mathrm{O}^{\oplus} \mathrm{H}, 2 \mathrm{O}^{\ominus} \mathrm{H}$ & $56,82,102$ & 53.3 & & 18.4 \\
\hline 117 & $\mathrm{C}^{\ominus} \equiv \mathrm{O}^{\oplus}, \mathrm{H}_{3} \mathrm{O}^{\oplus}, \mathrm{O}^{\ominus} \mathrm{H}$ & 54,87 & 7.6 & & 5.1 \\
\hline 118 & $\mathrm{C}^{\ominus}\left(=\mathrm{O}^{\oplus} \mathrm{H}\right) \mathrm{O}^{\oplus} \mathrm{H}_{2}, \mathrm{O}^{\ominus} \mathrm{H}$ & $57,59,83,85$ & 65.7 & & 42.2 \\
\hline 119 & $\mathrm{C}^{\oplus}\left(\mathrm{O}^{\ominus}\right)_{3}, \mathrm{H}_{2}, 2 \mathrm{H}^{\oplus}$ & $60,72,128$ & 17.7 & 7.1 & \\
\hline 120 & $\mathrm{HC}^{\oplus}\left(\mathrm{O}^{\ominus}\right)_{2}, \mathrm{O}^{\ominus} \mathrm{H}, 2 \mathrm{H}^{\oplus}$ & $61,63,73,75,106$ & 257.8 & & 98.4 \\
\hline 121 & $\mathrm{C} \ominus(\mathrm{O} \ominus)_{2} \mathrm{O}^{\oplus} \mathrm{H}_{2}, 2 \mathrm{H}^{\oplus}$ & $62,78,89,94$ & 111.3 & & 55.6 \\
\hline 122 & $\mathrm{C}^{\oplus}\left(\mathrm{O}^{\ominus}\right)_{2} \mathrm{O}^{\oplus} \mathrm{H}_{2}, \mathrm{H}^{\oplus}, \mathrm{H}^{\ominus}$ & $65,79,90,94,109$ & 235.5 & 38.8 & \\
\hline 123 & $\mathrm{C}^{\ominus}\left(=\mathrm{O}^{\oplus} \mathrm{H}\right) \mathrm{O}^{\ominus}, \mathrm{O}^{\ominus} \mathrm{H}, 2 \mathrm{H}^{\oplus}$ & $64,80,85,91,97$ & 158.2 & & 68.7 \\
\hline 124 & $\mathrm{C}^{\oplus}(=\mathrm{O} \oplus \mathrm{H}) \mathrm{O} \ominus, \mathrm{O} \ominus \mathrm{H}, \mathrm{H}^{\oplus}, \mathrm{H}^{\ominus}$ & $66,69,81,86,92,97,111$ & 481.6 & 118.1 & \\
\hline 125 & $\mathrm{C} \equiv \mathrm{O}^{\oplus}, 2 \mathrm{O} \ominus \mathrm{H}, 2 \mathrm{H}^{\oplus}$ & $68,87,101$ & 48.6 & & 22.0 \\
\hline 126 & $\mathrm{C}^{\oplus} \equiv \mathrm{O}^{\oplus}, 2 \mathrm{O}^{\ominus} \mathrm{H}, \mathrm{H}^{\oplus}, \mathrm{H}^{\ominus}$ & $70,88,101,115$ & 143.5 & 19.3 & \\
\hline 127 & $\mathrm{C}^{\ominus}\left(\mathrm{O}^{\ominus}\right)_{3}, 4 \mathrm{H}^{\oplus}$ & 89,105 & 15.6 & & 8.7 \\
\hline 128 & $\mathrm{C}^{\oplus}\left(\mathrm{O}^{\ominus}\right)_{3}, 3 \mathrm{H}^{\oplus}, \mathrm{H}^{\ominus}$ & $71,90,105,119$ & 102.6 & 11.1 & \\
\hline
\end{tabular}

